\title{
Aggregation Phenomena and Electromagnetic Amplification Properties in Silver Nanoparticles Joined Through Highly Conjugated Carbon Chains
}

\author{
Giuseppe Compagnini ${ }^{*},{ }^{1}$, Gabriele C. Messina ${ }^{1}$, Luisa D’Urso ${ }^{1}$, Elena Messina ${ }^{1}$, Marco G. Sinatra ${ }^{1}$, \\ Orazio Puglisi ${ }^{1}$, Massimo Zimbone ${ }^{2}$ \\ ${ }^{I}$ Dipartimento di Scienze Chimiche, Università degli Studi di Catania, Viale Andrea Doria 6 Catania 95125, Italy \\ ${ }^{2}$ Dipartimento di Fisica e Astronomia, Università di Catania, Viale Andrea Doria 6 Catania 95125, Italy
}

\begin{abstract}
Silver colloids prepared by laser ablation in water have been linked using sp-hybridized carbon chains thus obtaining a plasmonic transfer between the metallic nanoparticles. It has been found that the state of the metallic surface is of fundamental importance for the plasmonic transfer. The role of the carbon $\pi$ - electron system has been considered in order to justify the electromagnetic field amplification and the observed surface enhanced Raman signals. These findings open the possibility to control the electronic transfer between different metallic nanostructures for the application in future plasmonic devices.
\end{abstract}

Keywords: Metal nanoparticles, plasmonics, SERS effect, laser ablation.

\section{INTRODUCTION}

Beside nanoscale welding [1], metallic nanoparticles can be joined by different molecular entities thus obtaining collective properties without their direct contact as in the case of plasmon coupling [2].

A metal nanoparticle supports localized surface plasmons, which are associated with the collective oscillation of the conductive electrons. The surrounding electromagnetic field, excited near the plasmon resonance can extend quite some distance away [2], so that a compelling analogy exists between plasmon resonances of nanoparticle assemblies and wave functions of simple atoms and molecules. The strong interactions of plasmons in nanoparticle assemblies can render possible many useful applications including surface-enhanced spectroscopies, nano-antennas, and biochemical sensors [3-5]. In most of them the state of the nanoparticles surface is of paramount importance.

In the last few years pulsed laser ablation in liquids (PLAL) has been considered one of the most interesting techniques to obtain metal colloids with controlled size and shape in a single step [6]. This technique has also the advantage to be environmentally friendly and to produce sols which are free of unwanted by-products. Moreover it has been observed that the metal nanoparticles produced by PLAL present negative charges on the surface, that stabilize the colloidal solution due to the presence of electrostatic repulsion forces, and therefore do not require the addition of stabilizers or binders in order to control aggregation process. It was demonstrated that the negative charge is induced by the presence of oxidized metal atoms on the surface

\footnotetext{
*Address correspondence to this author at the Dipartimento di Scienze Chimiche, Università degli Studi di Catania, Viale Andrea Doria 6 Catania 95125, Italy; Tel: +39 095 7385077; Fax: +39 095 580138;

E-mail: gcompagnini@unict.it
}

of nanoparticle [7]. Furthermore, several studies conducted by Liao et al. [8], associated aggregation phenomena of noble metal nanoparticles in water to strong dipole/dipole interactions generated by an asymmetric charge distribution onto the cluster surface. Changes in the surface charge state and therefore on aggregation phenomena can be photoinduced by laser irradiation at different wavelenghths [9].

This work intends to investigate the interaction between silver clusters prepared by PLAL in different ways and freshly prepared Linear Carbon Chains (LCCs, frequently termed also polyynes or carbon nanowires), demonstrating that polyynes can act as plasmonic linkers between the nanoparticles. The presence of conjugated bonds in the LCCs with a high $\pi$-electron density ensures the electron transfer with a high efficiency in all those cases in which carbon is bonded onto a oxide free metal surface. Extended Mie theory will also strengthen the results supporting a view in which carbon connected silver nanoaprticles behave like a single aggregate with its own plasmon confinement.

\section{EXPERIMENTAL SECTION}

Silver colloidal sols were prepared using laser ablation in water. A Nd:YAG laser beam (5 nsec pulse duration and 10 $\mathrm{Hz}$ repetition rate) was focused through a lens on a pure metal target submerged in Millipore grade water $(5 \mathrm{~mL})$. Both the first $(1064 \mathrm{~nm})$ and the second harmonic $(532 \mathrm{~nm})$ radiations have been used. It is now well established that the plasma generated during the ablation process contains different ablated species as well as ions and radicals coming from water and all of them contribute to the formation of the final suspension $[10,11]$.

Polyynes were produced by igniting an arc discharge between two graphite electrodes in water using a procedure similar to that already reported by Cataldo [12]. Specifically, two cylindrical graphite electrodes with a diameter of about $6 \mathrm{~mm}$ were connected to a DC supplier. The voltage was set at $30 \mathrm{~V}$ and the measured current was $10 \mathrm{~A}$ for about $10 \mathrm{~min}$. 
After arcing, the obtained liquid has been filtered with a 0.22 micron filter (Millipore Millex in PES) to remove the large amount of carbon dust formed during the discharge process. In a series of papers Cataldo [13] has shown that such a procedure leads to the formation of polyynes with a concentration up to $10^{-3} \mathrm{M}$ and traces of few polycyclic aromatic hydrocarbons. In order to control the polyyne concentration, specific absorption measurements in the range 190-300 $\mathrm{nm}$ have been performed and the data have been analyzed using known molar extinction coefficients taken from literature data [14]. The stability of the obtained solution has been already tested using either UV absorption and Raman spectroscopy, as reported elsewhere [15].

To obtain inorganic/organic hybrids, silver colloids and polyyne suspensions were prepared separately and immediately mixed. UV-Vis spectra were performed on as prepared silver colloids and on filtered aqueous suspension of LCCs with a Jasco V-650 spectrometer. Raman spectra of mixed systems were performed using two different laser sources: an Ar laser $(\lambda=514.5 \mathrm{~nm})$ and a He:Ne laser $(\lambda=632.8 \mathrm{~nm})$. All the Raman spectra were directly performed in liquid using a 10X optical objective (laser power density below $10 \mathrm{~mW}$ ) and a Jobin Yvon $450 \mathrm{~mm}$ focal length monochromator, equipped with a CCD camera detector cooled at $77 \mathrm{~K}$. Some drops of the as prepared colloids have been deposited onto a silicon substrate for XPS measurements. These were obtained using an AXIS-ULTRA spectrometer with a basic chamber pressure in the range of $10^{-9}$ Torr. The X-ray radiation was generated by an Al K $\alpha$ line decay $(1486 \mathrm{eV})$ at operating conditions of $10 \mathrm{KV}$ and $15 \mathrm{~mA}$. The emitted photoelectrons were analyzed with a hemispherical electron energy analyzer. The detailed spectra have been acquired with a resolution below $1 \mathrm{eV}$.

\section{RESULTS AND DISCUSSION}

\subsection{Plasmon Resonence and Surface State}

Fig. (1) reports the surface plasmon resonance for the as prepared silver colloids (red) and for the colloids after the interaction with the carbon chains (blue). In particular Fig. (1a) regards a colloid prepared using the $532 \mathrm{~nm}$ radiation (Ag@532), while in Fig. (1b) we report the behaviour in the case of silver clusters produced by ablation at $1064 \mathrm{~nm}$ (Ag@1064). The plasmon resonance spectra for both as prepared colloids appear very similar in terms of position and width; so the result are not influenced by the laser wavelength used for the ablation. Nevertheless while at 1064 $\mathrm{nm}$ the pulsed beam does not interact with already formed particles in water (at this wavelength the light absorption is negligible), at $532 \mathrm{~nm}$ it overlaps with the tail of the plasmonic signal, thus increasing locally the colloidal temperature [16]. Once the carbon chain solution is added to the sol, the two colloidal suspensions behave in a deeply different way. Slight variations were detected in the SPR for LCCs-Ag@532 samples. Only a red shift of $14 \mathrm{~nm}$ and a certain increase of the signal width is observed. On the contrary in LCCs-Ag@1064nm samples a second plasmon component appears at about $713 \mathrm{~nm}$, while the component at lower wavelength remains positioned at about $416 \mathrm{~nm}$. Several works reported in literature [17] consider the SPR splitting $\left(\lambda-\lambda_{0}\right)$ as indicative of a plasmon coupling among metal nanoparticles. An exponential decay is reported as function of the distance $(\mathrm{g})$ between two interacting nanoparticles of diameter $\mathrm{D}$. The so called plasmon ruler equation is reported below:

$\frac{\lambda-\lambda_{0}}{\lambda_{0}}=0.18 \exp ^{\left(\frac{-(g / D)}{0.23}\right)}$

It is also useful to define $\sigma$ as the $g / D$ ratio. This mathematical model is based on a simple dipole-dipole coupling approximation and is roughly valid for all kinds of metals. In our case, if we suppose that the average silver cluster diameter is $15 \mathrm{~nm}$ (see TEM image in the inset of Fig. 1a) and the average carbon chain length is $1.19 \mathrm{~nm}$ (corresponding to 8 carbon atoms/chain, detectable by Raman studies [18]), the value of $\sigma$ should result far below 1.05 which is considered as a limiting value for the appearance of a double resonance contribution. In this specific case the extended $\pi$ electron system of the carbon chain acts as a plasmonic linker.

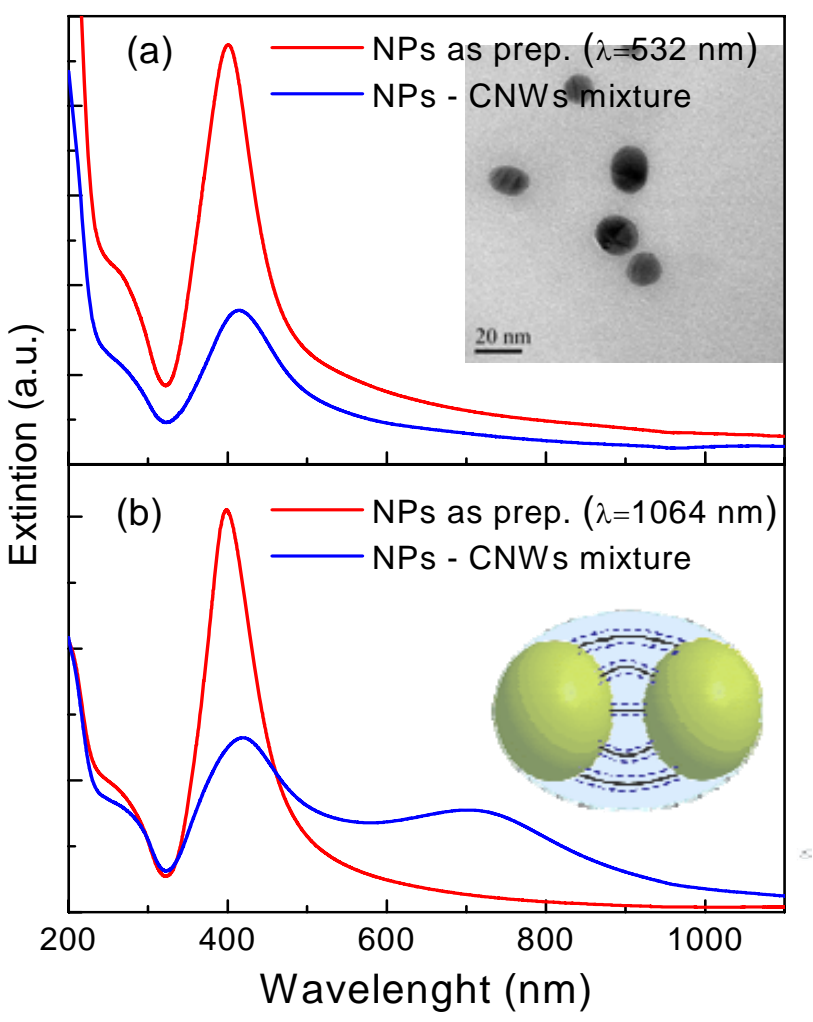

Fig. (1). Plasmon resonances for silver colloids in water before and after the interaction with carbon nanowires (CNWs). The colloidal solutions have been prepared using either a visible (a) or an infrared (b) radiation during the ablation procedure, as described in the text.

We have previously mentioned that the use of different laser wavelengths during the ablation process causes a different increase of the metallic particles temperature and a change of its surface chemical state. Considering that the surface state represents a key parameter to obtain a plasmonic connection, we performed some XPS analysis on all the available as prepared samples. The most important results are reported in Fig. (2). 


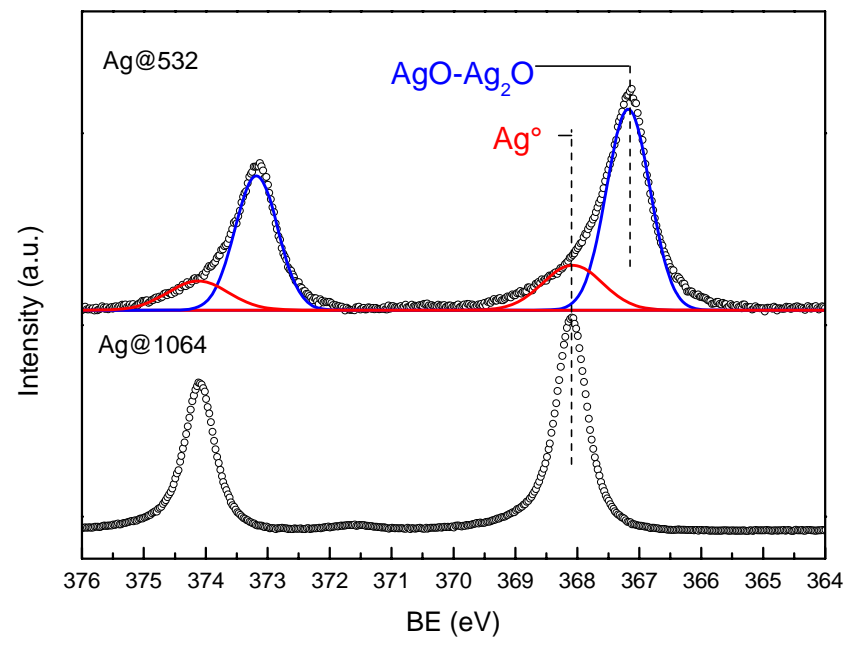

Fig. (2). XPS spectra in the region of the Ag3d core electrons indicating the presence of silver oxides species in those silver particles prepared by ablating the metal target with a $532 \mathrm{~nm}$ radiation.

Here Ag 3d core level spectra have been analyzed for the two Ag systems. XPS analysis clearly indicates that the Ag3d binding energies of $\mathrm{Ag} @$,532 aggregates are located at lower $(0.93 \mathrm{eV})$ energy respect to the Ag@1064 peaks. Such a shift indicates the presence of an oxide shell (AgO and/or $\mathrm{Ag}_{2} \mathrm{O}$ ) at the particle surface which is formed during or immediately after its growth.

In the case of $\mathrm{Ag} @ 1064 \mathrm{~nm}$ the amount of oxide is negligible and the surface can be considered "oxide free". Fig. (2) also shows the position of metallic silver and the increase of the line width for the oxide signal, in agreement with literature data in which $\mathrm{AgO}$ is considered a mixed oxide with the presence of both $\mathrm{Ag}^{+}$and $\mathrm{Ag}^{3+}$ ions [19]. In view of the XPS results we suggest that the oxide layer prevents the chemisorptions of the linear carbon chains thus preventing the phonon coupling. At this stage we can suppose that polyyne molecules bridge the metal nanoparticles when the colloids are obtained by ablation at $1064 \mathrm{~nm}$ while if a $532 \mathrm{~nm}$ radiation is employed the carbon wires tend to self-assemble around aggregated nanoparticles. Following DFT calculations [20] when the nanowires are put in contact with metallic silver (1064 $\mathrm{nm}$ ablation case) the terminal carbons interact with $\mathrm{Ag}$ atoms giving rise to a covalent bond which is also responsible for the formation of longer polyynic linear chains assisted by the adsorption of shorter ones at the nanoparticle surface.

\subsection{Simulations of the Observed Plasmon Resonences}

The entire plasmon resonance spectrum can be simulated using a classical Mie approach for a large number of metal nanoparticles [21]. In this respect, it is to be considered that when a small spherical metallic nanoparticle is irradiated by light, the oscillating electric field induces the conduction electrons to oscillate coherently. Thus the electron cloud is displaced relative to the nuclei and a restoring force arises from Coulomb attraction between electrons and nuclei that results in oscillation of the electron cloud relative to the nuclear framework.

The oscillation frequency is determined by four factors: the density of electrons, the effective electron mass, and the shape and size of the charge distribution. Moreover for a metals like silver, the oscillation frequency is also influenced by other electrons such as those in d-orbitals, and this prevents the oscillation frequency from being easily calculated using electronic structure calculations.

The simplest method to take account of plasmon resonance is the dipole approximation but it is strictly valid only for small particles; for larger particles, the dipole approximation is not justified due to significant phasechanges of the driving field over the particle volume, and higher modes of plasmon excitation have to be considered, such as the quadrupole mode where half of the electron cloud moves parallel to the applied field and half moves antiparallel.

For larger particles rigorous electrodynamic approach is required and it is necessary to use the classical Mie theory.

In the case of a particle much smaller than the wavelength of light it is generally sufficient to consider a dipole plasmon approach. In fact, under these circumstances, the electric field of the light can be taken to be constant, and the interaction is governed by electrostatics rather than electrodynamics. This is often called the quasistatic approximation, since the wavelength-dependent dielectric constant of the metal particle $(\varepsilon)$ and of the surrounding medium $\left(\varepsilon_{\mathrm{m}}\right)$ are used. The polarizability of a sphere of radius $R$ is given by:

$\alpha=R^{3} \frac{\varepsilon-\varepsilon_{m}}{\varepsilon+2 \varepsilon_{m}}$

If the particle cannot be considered "small" enough multipolar terms have to be accounted following the complete Mie (spherical) or Mie-Gans (ellipsoidal) theory. For instance it is necessary to introduce a quadrupolar terms such as:

$$
\beta=R^{5} \frac{\varepsilon-\varepsilon_{m}}{\varepsilon+3 / 2 \varepsilon_{m}}
$$

Following this last approach we have simulated the spectra, already reported in Fig. (1b), by using the complete Mie theory for spherical particles with a bulk silver dielectric constant.

The results of the simulations (lines) together with the experimental spectra (dots) are reported in Fig. (3). Note that simulations have been performed considering different sphere radii from 10 to $90 \mathrm{~nm}$. In this way the contribution of the multipolar terms results much more important by increasing the nanoparticle size.

In particular, Fig. (3) shows that for small particles (10$18 \mathrm{~nm}$ ) appear a unique resonance structure that shifts (to a larger wavelength) and spreads the FWHM increasing the radius. For larger size $(30-90 \mathrm{~nm})$, one or two other resonances appear showing the quadrupole and higher order resonance.

A strict comparison between the experimental data and the simulations indicates once again that the overall extinction spectra of silver clusters connected by carbon nanowires can be considered as the result of an extended (spherical) network of plasmon connected metal nanoparticles free of oxide shells. Fig. (3b) shows that is 
necessary to consider resonances extended to a $90 \mathrm{~nm}$ global entity (at least three of the as prepared nanoparticles) in order to simulate the experimental plasmon resonance spectrum.
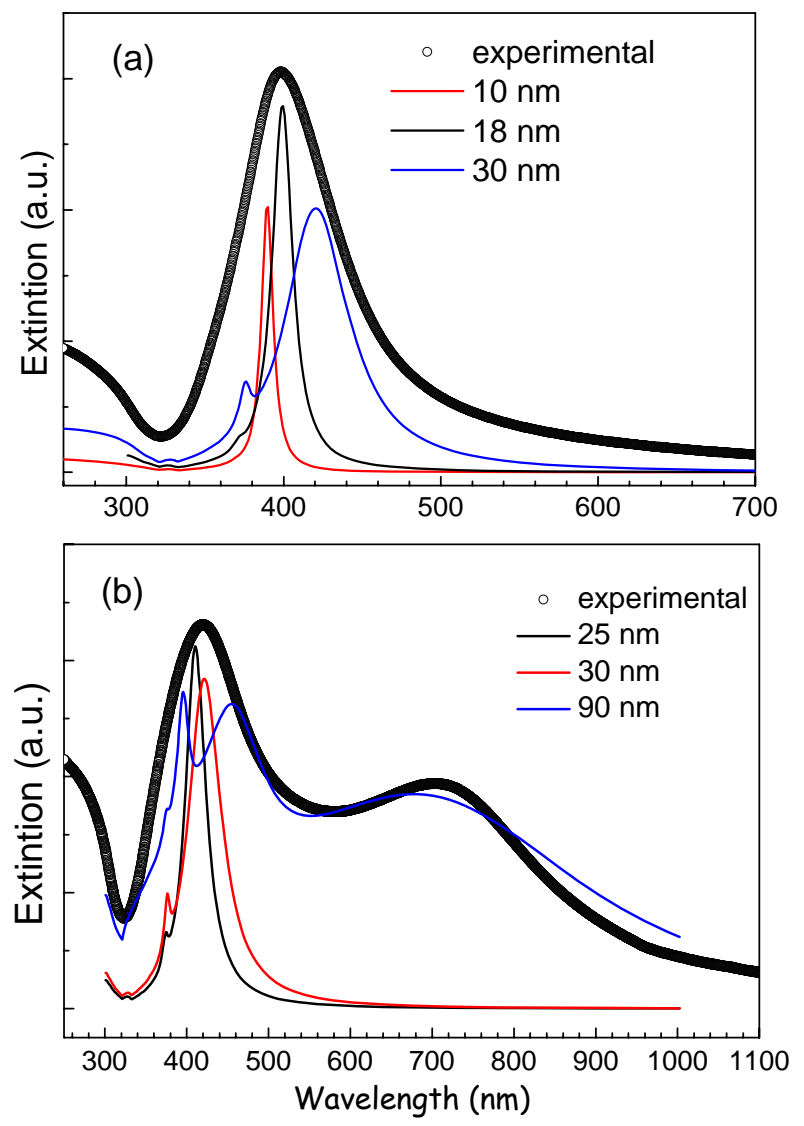

Fig. (3). Plasmon resonances of silver nanoparticles with (a) and without (b) the oxide shell after the interaction with carbon nanowires. The plasmon resonances are compared with simulated spectra obtained with a Mie approach in a multipolar expansion approximation. The shown simulations are obtained using different particle size in a spherical configuration.

\subsection{SERS Implications}

One of the most interesting applications of metal nanoparticles is related to their use for the so called "Surface Enhanced Raman Effect", whose first observation dates back to the '70s. An area of great interest in the SERS community concerns the observation of single-molecule SERS (SMSERS) [22]. This aspect, which was originally developed in 1997, has proven to be of fundamental interest due to the nominal enhancement factor above $10^{13}$.

The best SERS magnification is obtained once the laser excitation wavelength is close to the plasmon resonance of the metallic nanoparticles [23] and when the geometrical distribution of the particles produces an intense local electric field [24]. This last condition is realized when the molecular specie under investigation is positioned in between nearly touching particles.

In accordance with Raman spectroscopic studies on spbonded carbon species [25], it is possible to observe clear
SERS signals detected in the $1800-2150 \mathrm{~cm}^{-1}$ region when the carbon chains have a number of carbon atoms between 6 and 20. In addition to the sp vibrational features a broad band located between 1000 and $1700 \mathrm{~cm}^{-1}$ is generally detected, coming from highly disordered graphite-like structures [26].

These signals are evident in Fig. (4) in those cases where the carbon nanowires are plasmonically connected with silver nanoparticles (joined NPs). In fact in these cases either the $514 \mathrm{~nm}$ radiation and the 633 ones overlaps with the surface plasmon resonances already shown in Fig. (1). Then, even though both mixed systems present an electromagnetic field enhancement [27], only LCCs-Ag@1064 presents a charge transfer enhancement and signals of one order of magnitude larger.

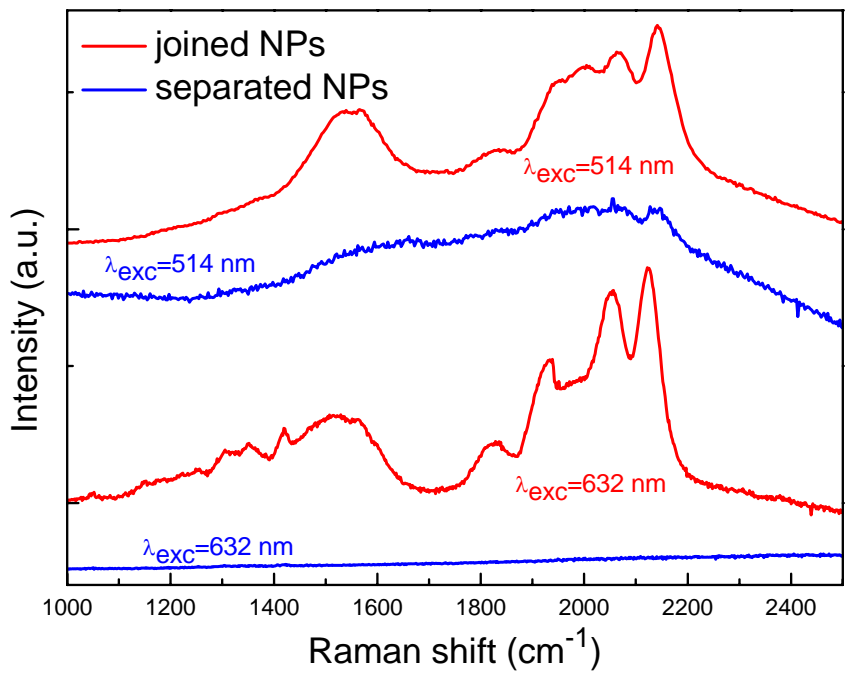

Fig. (4). Surface enhanced Raman spectra exited with different wavelength in the case of colloidal solution with a single and a double resonance.

\section{CONCLUSIONS}

This paper has shown that it is possible to join metallic nanoparticles, inducing a collective plasmon resonance, using carbon wires with high $\pi$ electron density (polyynes).

This effect can be obtained only in those cases in which the particles' surface is free of contaminations and oxides. For this reason pulsed laser ablation in water has been used with specific experimental parameter in order to produce suitable the silver colloids.

Specific SERS results have been reported which straighten these considerations and envisage future applications.

\section{REFERENCES}

[1] Lu Y, Huang JY, Wang C, Sun S, Lou J. Cold welding of ultrathin gold nanowires. Nat Nanotech 2010; 5: 218-24.

[2] Hentschel M, Saliba M, Vogelgesang R, Giessen H, Alivisatos AP, Liu N. Transition from isolated to collective modes in plasmonic oligomers. Nano Lett 2010; 10: 2721-6.

[3] Compagnini G, Galati C, Pignataro S. Distance dependence of surface enhanced raman scattering probed by alkanethiol self assembled monolayers. Phys Chem Chem Phys 1999; 1: 2351-3.

[4] Yuen JM, Shah NC, Walsh JT, Glucksberg MR, Van Duyne RP. Transcutaneous glucose sensing by surface-enhanced spatially offset raman spectroscopy in a rat model. Anal Chem 2010; 82: 8382-5. 
[5] Wissert MD, Ilin KS, Siegel M, Lemmer U, Eisler HJ. Coupled Nanoantenna Plasmon Resonance Spectra from Two-Photon Laser Excitation. Nano Lett 2010; 10: 4161-5.

[6] Compagnini G, Scalisi AA, Puglisi O. Synthesis of gold colloids by laser ablation in thiol-alkane solutions. J Mat Res 2004; 19: 27952798.

[7] Mafune SPIE Newsroom 10.1117/2.1200911.1839.

[8] Liao J, Zhang Y, Yu W, Ge C, Liu J, Gu N. Linear aggregation of gold nanoparticles in ethanol. Colloids Surf A 2003; 223: 177-83.

[9] Tsuji T, Iryo K, Watanabe N, Tsuji M. Preparation of silver nanoparticles by laser ablation in solution: influence of laser wavelength on particle size. Appl Surf Sci 2002; 202: 80-5.

[10] Vogel A, Noack J, Nahen K, et al. Energy balance of optical breakdown in water at nanosecond to femtosecond time scales. Appl Phys B 1999; 68: 271-80.

[11] Compagnini G, Messina E, Puglisi O, Nicolosi V. Laser synthesis of $\mathrm{Au} / \mathrm{Ag}$ colloidal nano-alloys: exploring the optical properties for an accurate analysis. Appl Surf Sci 2007; 254: 1007-011.

[12] Cataldo F. Synthesis of polynes in a submerged electric arc in organic solvents. Carbon 2004; 42: 129-42.

[13] Cataldo F. Polyynes: Synthesis, properties, and applications. Taylor and Francis 2006.

[14] Eastmond R, Johnson TR, Walton DRM. Silylation as a protective method for terminal alkynes in oxidative couplings: A general synthesis of the parent polyynes $\mathrm{H}(\mathrm{C}=\mathrm{C})_{\mathrm{n}} \mathrm{H} \quad(\mathrm{n}=4-10,12)$. Tetrahedron 1972; 28: 4601-16.

[15] Compagnini G, Mita V, D’Urso L, Cataliotti RS, Puglisi O. Spectroscopic study of polyynes obtained by laser ablation in liquids. J Raman Spect 2008; 39: 177-81.

[16] Bruzzone S, Malvaldi M. Local field effects on laser-induced heating of metal nanoparticles. J Phys Chem C 2009; 113: 1580510.
[17] Reinhard BM, Siu M, Agarwal H, Alivisatos AP, Liphardt J. Calibration of dynamic molecular rulers based on plasmon coupling between gold nanoparticles. Nano Lett 2005; 5: 2246-52.

[18] Scuderi V, Scalese S, Bagiante S, Compagnini G, D’Urso L, Privitera V. Direct observation of the formation of linear C chain/carbon nanotube hybrid systems. Carbon 2009; 47: 2134-7.

[19] Cotton FA, Wilkinson G. Advanced Inorganic Chemistry, 5th ed. Wiley-Interscience: New York 1988.

[20] Lucotti A, Tommasini M, Del Zoppo M, et al. Raman and SERS investigation of isolated sp carbon chains. Chem Phys Lett 2006; 417: 78-82.

[21] Kreibig U, Vollmer M. Optical properties of metal clusters. Springer: New York 1994.

[22] Kneipp K, Wang Y, Kneipp H, et al. Single molecule detection using surface-enhanced raman scattering. Phys Rev Lett 1997; 78: 1667-70.

[23] McFarland AD, Young MA, Dieringer JA, Van Duyne RP. wavelengthscanned surface-enhanced raman excitation spectroscopy. J Phys Chem B 2005; 109:11279-85.

[24] Camden JP, Dieringer JA, Wang Y, et al. Probing the structure of single-molecule surface-enhanced raman scattering hot spots. J Am Chem Soc 2008; 130: 12616-7.

[25] D'Urso L, Compagnini G, Puglisi O. sp/sp $\mathrm{sp}^{2}$ bonding ratio in sp rich amorphous carbon thin films. Carbon 2006; 44: 2093-5.

[26] Compagnini G, Baratta G. Polarized Raman spectroscopy in ion irradiated graphite. Appl Phys Lett 1992; 61: 1796-8.

[27] Park W, Kim WZ. Charge transfer enhancement in the SERS of a single molecule. Nano Lett 2010; 10: 4040-48. 